\title{
LAS PRIMAVERAS ÁRABES: LA INFLUENCIA DE QATAR Y SUS RELACIONES CON LOS ESTADOS DEL GOLFO
}

\author{
Alberto Priego ${ }^{1}$ \\ UNISCI / Universidad Pontificia Comillas
}

\begin{abstract}
Resumen:
Las Primaveras Árabes han supuesto el fin de la influencia Occidental en el Norte de África y Oriente Medio. Hasta el 2011 la UE y Estados Unidos eran actores influyentes en la región. El fracaso de las Primaveras Árabes ha permitido la entrada de otros actores, concretamente de los estados del Golfo Pérsico. Qatar y Arabia Saudí han visto la oportunidad de convertirse en potencias regionales con capacidad de influir. Sin embargo, la ventana de oportunidad ha provocado una competición entre Doha y Riad que pugnan por convertirse en los líderes regionales.
\end{abstract}

Palabras clave: Qatar, Arabia Saudí, Primavera Árabe, Unión Europea, Hermanos Musulmanes.

Title in English: "The Arab Springs: Qatar's Influence and its Relations with the Gulf States"

\begin{abstract}
:
The Arab Springs have been the end of the Western influence in North Africa and the Middle East. Until 2011 the EU and the United States were strong influential actors in the region. The failure of the Arab Spring has allowed the entry of other actors, particularly the Gulf states. Qatar and Saudi Arabia have seen the opportunity to become regional powers capable of exerting influence in this area. However, the window of opportunity has led to a competition between Doha and Riyadh, both wanting to become the regional leaders.
\end{abstract}

Keywords: Qatar, Saudi Arabia, Arab Spring, European Union, Muslim Brotherhood.

Copyright (C) UNISCI, 2015.

Las opiniones expresadas en estos artículos son propias de sus autores, y no reflejan necesariamente la opinion de UNISCI. The views expressed in these articles are those of the authors, and do not necessarily reflect the views of UNISCI.

\footnotetext{
${ }^{1}$ El Dr. Alberto Priego es profesor propio adjunto en el Área de Ciencia Política (Departamento de Relaciones Internacionales) en la Universidad Pontificia Comillas (ICADE), ha sido investigador invitado en la School of Oriental and African Studies (The University of London), es investigador senior de UNISCI y miembro del Foro Hispano-Argelino.

E-Mail: apriego@upcomilllas.es.

http://dx.doi.org/10.5209/rev_RUNI.2015.n39.5180u
} 


\section{Introducción}

Las Primaveras Árabes han emergido como un fenómeno sin precedentes que ha supuesto el fin del monopolio Occidental en Oriente Medio y Norte de África. La Nueva Política de Vecindad de la Unión Europea se ha mostrado como uno de los mayores fracasos del proyecto europeo. En el flanco éste, Rusia se ha aprovechado de las diferencias surgidas entre los miembros de la UE para reocupar lo que considera "su espacio". En el flanco sur, la situación no ha sido muy distinta. Líderes árabes, que hasta ahora eran apoyados por Occidente, han sido depuestos por movimientos populares de corte islamista. Los nuevos gobernantes han favorecido la entrada de otros actores, esencialmente estados del Golfo, que han aparecido en la región ocupando el espacio que no han podido llenar por motivos económicos y de afinidad cultural y religiosa tanto la UE como los Estados Unidos, si bien queda por estudiar el posible visto bueno estadounidense en todas estas actuaciones o una supervisión/aceptación. Quizás la forma más gráfica de entender este fenómeno la encontremos en uno de los principios que ha guiado la política exterior de Qatar "Árabes para solucionar los problemas de los árabes".

Por ello, podemos afirmar las Revoluciones Árabes han supuesto además de un cambio de relaciones entre Europa y Oriente Medio, un cambio en las relaciones entre los estados del Golfo Pérsico entre sí. En buena medida, se han enfrentado dos posiciones claramente contrapuestas.

- La primera - pro status quo- ha sido liderada por Arabia Saudí. A estas posiciones se han unido otros actores regionales como Kuwait, Emiratos Árabes Unidos ${ }^{2}$ y Bahréin. Los defensores de esta línea han abogado por el mantenimiento del orden establecido y su mayor éxito ha sido la restauración del orden militar en Egipto tras la victoria de los Hermanos Musulmanes en las elecciones.

- La segunda posición -revisionista- estuvo defendida por Q $\operatorname{atar}^{3}$ y su principal particularidad fue la defensa de una transformación estructural del mundo árabe apoyándose para ello en las redes de los Hermanos Musulmanes que se erigieron como la fuerza del cambio. Qatar apostó fuertemente sobre todo en Libia y Egipto aunque, tras sendos fracasos, ahora, con un perfil más bajo, busca refugio en el caso tunecino.

Debido a una serie de circunstancias más o menos inesperadas -el cambio de Emir en Qatar ${ }^{4}$, la emergencia del ISIS o el citado fracaso de la vía de la hermandad-parece que los partidarios de la primera opción estén ganando la batalla a los segundos. Aunque no es menos cierto que las posiciones de Arabia Saudí tras la coronación del rey Salman han cambiado considerablemente.

La hipótesis de partida de este trabajo es la siguiente: Las Revoluciones Árabes deben ser entendidas como una lucha por el poderio regional entre Qatar y Arabia Saudi, dos estados que pugnan por convertirse en el actor regional de referencia en el mundo árabe. Para ello vamos a analizar el rol de Qatar en las Primaveras Árabes con especial atención a Libia, Egipto, Siria y en menor medida Túnez. En todos estos casos, se aprecia de forma clara la ya mencionada rivalidad entre Qatar y Arabia Saudí. Mientras que Doha ha apostado por un

\footnotetext{
${ }^{2}$ A partir de ahora EAU.

${ }^{3}$ Según la RAE en Ortografía de la lengua española de 2010 las formas Qatar y Catar son igualmente válidas por lo que en este trabajo se usará la grafía con Q por ser más aceptada a nivel internacional.

${ }^{4}$ La llegada de Nuevo Emir ha supuesto un acercamiento a Arabia Saudí así como una mayor concentración en los asuntos internos.

5 "Qatar's foreign policy decisions and their repercussions have been tightly connected to its long-standing rivalry with Saudi Arabia over the two Gulf countries' degree of regional influence" Khatib, Lina: "Qatar and the Recalibration of Power in the Gulf", Carnegie Middle East Center, 11 September 2014.
} 
trasvase de poder a nuevos grupos, Riad prefiere el mantenimiento del status quo ${ }^{6}$, aunque esta afirmación está sujeta a matices. Ante la incertidumbre provocada por el cambio, Riad, ha apostado por grupos más cercanos al salafismo ${ }^{7}$ mientras que Qatar ha apostado por la hermandad. Quizás, quien mejor ha reflejado la política exterior de Qatar sea Lina Khatib cuando afirmó que Doha buscaba "maintain internal and regional (gulf) stability while claiming wider political influence ${ }^{8 "}$

El artículo se va a articular de la siguiente manera: En primer lugar vamos a realizar un análisis de los fundamentos de la política exterior de Qatar, por ser éste el actor más activo de la Primavera Árabe. Dentro de la acción de Qatar se van a analizar cuatro casos especialmente significativos: Túnez, Libia, Egipto y Siria. En todos ellos, además de la acción directa de Qatar se va a realizar una comparación con la influencia o contra-influencia saudí para demostrar nuestra hipóstesis. Para lograr una correcta aplicación de método comparado hemos seleccionado los siguientes aspectos que serán analizados en los cuatro casos de estudio: apoyo político-diplomático, la asistencia económica y la ayuda militar. Al final del trabajo demostraremos que la hipótesis de partida, la pugna entre Doha y Riad en el marco de las Primaveras Árabes, se cumple.

\section{Los fundamentos de la política exterior de Qatar}

Qatar, es un estado de pequeñas dimensiones $(11.586 \mathrm{Km} 2)$ con una población de poco más de un cuarto de millón de nacionales. Debido a estas características su posición internacional ha sido tradicionalmente muy modesta. Además, debido a sus condicionantes geográficos y, sobre todo, a la voracidad de estados vecinos (Irán y Arabia Saudí) la búsqueda de seguridad ${ }^{9}$ ha sido uno de los aspectos que han condicionado su política exterior. Por ello, Qatar se ha visto obligado a buscar métodos para garantizar su supervivencia y con tal fin no ha dudado en utilizar para dicho fin todos sus recursos disponibles. Entre todos sus esfuerzos podemos destacar dos estrategias que buscaban mejorar su posición internacional y sobre todo garantizar su seguridad $^{10}$ :

1) La búsqueda de un "Hermano" o un estado mayor. Doha siempre ha tenido la necesidad contar con un estado protector "qaim maqam" que se encargara de garantizar su supervivencia. La existencia de gigantes regionales como Irán, Irak y Arabia Saudí favoreció esta política. En el siglo XIX este papel fue ejercido por el Reino Unido y a raíz de la independencia de Qatar fue Estados Unidos ${ }^{11}$ quien se ocupó de dicha tarea.

\footnotetext{
6 "Doha systematically favors the Brotherhood, whereas Riyadh actively shuns and resists its rising influence" Haykel, Bernard: "Saudi Arabia and Qatar in a Time of Revolution" Gulf Analysis Paper (CSIS), February 2013, en http://csis.org/files/publication/130219_Haykel_SaudiQatar_GulfAnalysis.pdf.

7 "Doha Believed the moderate form of Islamism represented by the Brotherhood was a safer bet than the more reactionary Salafi groups supported by Saudi Arabia" Echagüe, Ana: "Emboldened yet vulnerable: the changing foreign policies of Qatar and Saudi Arabia" FRIDE-Working paper n 123 (julio 2014), p. 8, en http://fride.org/download/WP 123 Emboldened yet_vulnerable.pdf.

${ }^{8}$ Khatib, Lina: "Qatar's involvement in Libya: A delicate balance" World Peace Foundation, no 7 (enero 2013), en http://sites.tufts.edu/reinventingpeace/2013/01/07/qatars-involvement-in-libya-a-delicate-balance/.

9 "After the British left in 1971 it became apparent that Qatar could not defend itself as a country" Roberts, David: "Understanding Qatar's Foreign Policy Objective" Mediterranean Politics, vol. 17, nº. 2, (July 2012), p. 235.

10 "Preserving Qatar's security is the central tenet of Emir Hamad bin Khalifah al-Thani's vision" Roberts, David: "Qatar: domestic quietism, elite adventurism" Gulf Analysis-ECFR, abril 2013, p. 10.

11 "after 1990 and the Iraqi invasion of Kuwait, Qatar took his opportunity to sign basing agreements with America in 1992" Véase Roberts, David, op. cit., p. 235.
} 
De hecho, a día de hoy Estados Unidos posee algunas instalaciones militares en el emirato.

2) Alcanzar una posición única en la Comunidad Internacional: Como consecuencia de la invasión de Kuwait por parte de Sadam Hussein, Qatar comenzó a pensar que si bien la protección del "hermano mayor" era necesaria, ésta no era suficiente. Con la llegada al poder del Emir Hamad bin Khalifa Al Thani, Qatar inició una política exterior basada en un incremento exponencial de su notoriedad internacional así como en el desarrollo de un rol diferenciador respecto del resto de estados de la comunidad internacional. En este contexto destacan sus dotes de mediador en conflictos tales como Sudán del Sur, Yemen o Palestina. Así, Qatar ha buscado convertirse en un actor necesario que cumple una función muy particular que nadie más puede llevar a cabo. Qatar, asimismo, utilizará redes Salafistas/Wahhabistas, junto con Arabia Saudita y otros estados del Golfo, para la financiación de grupos religiosos que acabarán pudriendo determinados estados, caso de estados del Sahel y, en otro orden, de Siria ${ }^{12}$. Esta especificidad fue entendida por Doha como una protección especial frente a los gigantes regionales que le rodeaban.

\subsection{La religión como un elemento clave en la política exterior}

Uno de los aspectos fundamentales de la proyección exterior de Qatar ha sido sin lugar a dudas, la religión. Qatar puede ser definido como un Estado que sigue la tradición Wahabí, con una clara prevalencia de la escuela de jurisprudencia Hanbalí. De hecho, la familia Al Thani procede del mismo grupo tribal (the Bani Tamim) que el fundador del Wahabismo, Mohammed ibn Abd al-Wahhab. Sin embargo, aunque muchos de los postulados wahabistas se aplican en el país el Wahabismo ha sido percibido por los Al-Thani como una forma de dominación saudí. Es quizás por ello que Qatar ha buscado otros modelos político-religiosos en los que apoyarse ${ }^{13}$ y a los que parecerse, evitando identificarse con el Wahabismo por miedo a ser absorbido por Arabia Saudí.

En esta búsqueda los qataríes han encontrado el faro de los Hermanos Musulmanes quienes, desde hace décadas, han ostentado el liderazgo religioso en Qatar. Frente a la influencia saudí, los clérigos vinculados a los Hermanos Musulmanes presentan una gran virtud para la familia Al Thani: la no injerencia en los asuntos internos de Qatar ${ }^{14}$. Es precisamente esta característica la que ha inspirado la organización religiosa qatarí, frente al modelo modelo saudí. En el modelo qatarí, al contrario de lo que ocurre en Arabia Saudí, no hay una oficina del Gran Muftí evitando así que el poder religioso sirva como "contrapoder" al emir.

\footnotetext{
12 Véase un document del Unión Europea:"The involvement of Salafism/wahhabism in the support and supply of arms to rebel groups around the world" Directorate-General for External Policies of the Union, June 2013, en http://www.europarl.europa.eu/RegData/etudes/etudes/join/2013/457137/EXPOAFET ET\%282013\%29457137 EN.pdf.

13 "Wahhabism was thus a means by which the Al-Saud extended their influence over the other ruling tribes. In this regard, the dilemma for the rulers of Qatar was that although Wahhabism served a domestic purpose of shaping opinion and fostering legitimacy for the rulers, it also presented a challenge in that if the Ulama were to have too much power or influence they would conceivably align themselves with the Saudi state and thus pose a domestic challenge" Baskan, Birol and Wright, Steven: "Seeds of Change: Comparing State-Religion Relations in Qatar and Saudi Arabia" Arab Studies Quaterly, vol. 33, no. 2, (Spring 2011)

14 "The Brotherhood is barely involved in Qatari domestic affairs" Ahmed, Azem: "Qatar's ties with the Muslim Brotherhood Affect Entire Region" National, 18 mayo 2012, en

http://www.thenational.ae/thenationalconversation/comment/qatars-ties-with-the-muslim-brotherhood-affectentire-region.
} 
Son muchos los clérigos vinculados a la hermandad que desde hace décadas residen en Qatar. De hecho, la península se ha convertido en una especie de refugio para aquellos miembros de la hermandad que son perseguidos en sus países de origen. En concreto me estoy refiriendo a Egipto, Siria y en menor medida Arabia Saudí, EAU o Libia. Podemos destacar a miembros de la hermandad como Abdul-Badi Saqr, Ali al Sallabi o su hermano Ismail. Todos ellos, en su momento, encontraron asilo en Doha evitando así el castigo sus gobiernos ${ }^{15}$. Sin embargo, entre todos cabe destacar a Yusuf Al Qaradawi, quien, a pesar de haber nacido en Egipto, a día de hoy es considerado como la máxima autoridad religiosa del país. Este clérigo llegó a Qatar en los años 60 y desde entonces su figura no ha parado de crecer hasta convertirse, no sólo en un líder religioso del país sino también en una influyente personalidad. Como muestra de esta influencia, desde 1996 dirige y presenta -Sharia and Life- uno de los programa más populares de la televisión qatarí Al-Jazeera ${ }^{16}$.

La decisión de apostar por la hermandad es, ante todo, un intento de distinción en la Comunidad Internacional ya que ningún otro estado musulmán confía en los Hermanos Musulmanes ${ }^{17}$ como opción política de cambio. Es por ello que las Primaveras Árabes ha supuesto para Doha una oportunidad única para cumplir con los dos objetivos que hemos destacado anteriormente: por un lado incrementar su papel internacional distinguiéndose de los demás estados de su entorno, y por el otro aunque derivado de lo anterior, garantizar su supervivencia a nivel internacional. Estos hechos explican por qué Qatar ha tratado de impulsar en lugares como Yemen, Siria, Túnez, Libia y Egipto a partidos políticos vinculados a los Hermanos Musulmanes ${ }^{18}$ ya sea de forma directa o indirecta. Esta línea ha sido calificada como Daoud Boughezala como "la diplomatie du carnet de chèque ${ }^{19}$ " en una clara referencia a la financiación de estos partidos políticos vinculados a la hermandad.

Si bien es cierto que en un primer momento Doha pensó que las Primaveras Árabes podrían ser una opción de "democratización", no es menos cierto que la apuesta qatarí por la hermandad responde al ya mencionado ejercicio de pragmatismo. Es decir, aunque es cierto que el propio Emir defendió la tesis de que algunos islamistas podrían integrarse en la vida democrática $^{20}$, no es menos cierto que las Primaveras Árabes se plantearon como una ventana de oportunidad para Qatar para alcanzar sus propios fines. En una línea completamente diferente a la de, encontramos la visión saudí que no dudó en usar la fuerza -Peninsula Shieldpara "pacificar" Bahréin. En una línea similar Riad concedió asilo político a los presidentes derrocados (Saleh o Ben Alí) como muestra de desaprobación con las acciones adoptadas por Doha. Tal y como prueban estos dos ejemplos, Arabia Saudí se ha mostrado como un actor conservador y defensor del status quo, frente a Qatar que ha abogado una política revisionista introduciendo cambios sustantivos en el Comunidad Internacional. No obstante, como hemos mencionado anteriormente los cambios en las monarquías saudí y qatarí han provocado algunos cambios que posteriormente analizaremos con detalle.

\footnotetext{
15 "their relationship is based on a web of personal contacts established over the decades as Doha became host to a variety of actors, often of a Muslim Brotherhood persuasion" Roberts, David "Qatar: domestic quietism, elite adventurism" Gulf Analysis-ECFR, (abril 2013), p. 11.

${ }^{16}$ Roberts, David: "Qatar and the Brotherhood" Survival, vol. 56, no. 4, (August-September 2014), p. 25.

17 "Another key factor is personal politics with key Brotherhood figures", en Roberts, David B., p. 238. "Understanding Qatar's Foreign Policy Objective" Mediterranean Politics, Vol. 17, No. 2, July 2012.

18 "Qatar threw its weight behind the Brotherhood in transitioning countries, namely Egypt, Tunisia, Yemen and Libya", en Khatib, Lina: "Qatar and, op. cit.

${ }^{19}$ Boughezala, Daoud: "Tunisie. Ennadah la dernier carte de Qatar" Causeur, 23 noviembre 2013, en http://www.causeur.fr/tunisie-ennahda-la-derniere-carte-du-qatar-24880.html\#.

20 "I believe you will see this extremism transform into civilian life and civil society", Al Jazeera, 7 de septiembre de 2011 .
} 


\section{La acción de Qatar en las Primaveras Árabes}

Desde hace algunos años el mundo árabe está cambiando. Esencialmente el cambio viene marcado por la decadencia del modelo republicano-nacionalista (panarabista) y por la emergencia de las monarquías del golfo como referente del mundo árabe. De hecho las Primaveras Árabes no han hecho más que demostrar que el modelo pan-arabista defendido por Hosni Mubarak, Sadam Hussein o Bashar Al Assad ha llegado a su fin. Los gobernantes de estas repúblicas han basado su supervivencia en el paradigma "stability-first", postulándose frente a Occidente como la única alternativa frente a los islamistas. El mejor ejemplo de esta política quedó reflejada en una frase muy popular en el Túnez de Ben Alí: "Mieux vaut Ben Ali que Ben Laden".

Sin embargo, este modelo ha fracasado estrepitosamente y la "Guerra Fría Árabe ${ }^{21 "}$ entre las Monarquías del Golfo y las Repúblicas Nacionalistas se ha decantado del lado de los primeros. En este nuevo contexto, dos estados -Qatar y Arabia Saudí- se han estado disputando la posibilidad de convertirse en la nueva referencia para la joven población árabe que busca alternativas a la opción republicana panarabista.

En todo caso cabe preguntarse por qué se ha producido ese desencuentro entre unos líderes que en su momento fueron carismáticos y la población. La respuesta la debemos buscar en los altos niveles de desempleo (especialmente entre los jóvenes), en la corrupción y sobre todo la desconfianza de la población con un modelo político-económico fracasado. A esto le debemos unir la demonización de los líderes republicanos de opciones políticas no occidentales. Esencialmente me estoy refiriendo a grupos islamistas que tras el derrocamiento de estos regímenes y tras muchos años en la clandestinidad aparecieron como las únicas en posición de ilusionar a la población.

Cuadro 1: Situación en los estados de la Primavera Árabe (2011)

\begin{tabular}{|c|c|c|c|c|c|c|}
\hline Estado & Corrupción ${ }^{22}$ & Democracia $^{23}$ & $\begin{array}{l}\text { Desigualdad } \\
\text { GINI }^{24}\end{array}$ & $\begin{array}{l}\text { Freedom } \\
\text { Rating }^{25}\end{array}$ & $\begin{array}{l}\text { Derechos } \\
\text { Políticos }^{26}\end{array}$ & $\begin{array}{l}\text { Libertades } \\
\text { Civiles }^{27}\end{array}$ \\
\hline Túnez & $3.8 / 10$ & $\begin{array}{l}\text { No-Libre (FH) } \\
\text { Régimen } \\
\text { híbrido }\end{array}$ & $35.8(2008)$ & 6.0 & $7 / 7$ & $5 / 7$ \\
\hline Libia & $2 / 10$ & $\begin{array}{l}\text { No-Libre }(\mathrm{FH}) \\
\text { Régimen } \\
\text { híbrido }\end{array}$ & & 7.0 & $7 / 7$ & $7 / 7$ \\
\hline Egipto & $2.8 / 10$ & $\begin{array}{l}\text { No-Libre (FH) } \\
\text { Régimen } \\
\text { híbrido }\end{array}$ & $30.8(2008)$ & 5.5 & $6 / 7$ & $5 / 7$ \\
\hline
\end{tabular}

\footnotetext{
${ }^{21}$ Althani, Mohamed A. J., (2012): The Arab Spring \& the Gulf States, London, Profile Books, p. 9.

${ }^{22}$ Percepción de la corrupción donde 10 es muy limpio y 0 es corrupto, en http://www.transparency.org/cpi2011/results.

${ }^{23}$ The Economist Democracy Index 2012 distingue entre Full Democracies, Flawed Democracies, Hybrids Regimes y Authoritarian Regimes. Freedom House distingue entre Libres, Parcialmente Libres (3-5) y NoLibres (6-7).

${ }^{24}$ Fuente Banco Mundial.

${ }^{25}$ Fuente Freedom House. 7 es la peor calificación y 1 es la mejor.

${ }^{26}$ Fuente Freedom House. 7 es la peor calificación y 1 es la mejor.

${ }^{27}$ Fuente Freedom House. 7 es la peor calificación y 1 es la mejor.
} 


\begin{tabular}{|l|l|l|l|l|l|l|}
\hline Siria & $2.6 / 10$ & $\begin{array}{l}\text { No Libre (FH) } \\
\text { Régimen } \\
\text { Autoritario }\end{array}$ & 42.0 & 6.5 & $7 / 7$ & $6 / 7$ \\
\hline
\end{tabular}

Fuente: Elaboración propia.

Es en este el punto en el que entran en escena los estados del Golfo, especialmente Qatar que como hemos mencionado anteriormente durante décadas había ido tejiendo una red de conexiones con los Hermanos Musulmanes en todo el Mundo Árabe. Esta organización se erigió como la única opción "legítima" frente a otras que habían colaborado con los regímenes republicanos nacionalistas.

Además de gozar de esta aceptación popular, los Hermanos Musulmanes poseían "otra virtud", al haber sobrevivido en la clandestinidad eran los únicos que poseían una mínima organización para afrontar un proceso abierto y democrático como los que se avecinaban en el Norte de África tras la caída de los regímenes panarabistas. Este rasgo, la organización en la clandestinidad, es precisamente el que levanta mayores temores en Riad ya que la hermandad es el único grupo organizado que se ha opuesto a las acciones de la familia $\mathrm{Saud}^{28}$. No obstante, la llegada al trono del Rey Salman ha supuesto una reconfiguración de la relación con la hermandad. De hecho, durante este verano de 2015 se han producido encuentros de alto nivel con líderes como Rachid Ghannouchi ${ }^{29}$, Khaled Meshaal ${ }^{30}$ o Abdul Majeed Zindani ${ }^{31}$.

En todo caso, todavía es pronto para afirmar que esta mejora de las relaciones entre Riad y la Hermandad sea el inicio de una nueva era. Quizás este acercamiento bien podría estar propiciada por el temor a una mayor influencia irani ${ }^{32}$, especialmente ahora que va a disponer de más recursos para ejercer su influencia en aquellos lugar donde los Hermanos Musulmanes tienen o han tenido presencia (Túnez, Egipto, Yemen, Palestina etc...).

\subsection{Túnez}

El proceso de cambio en Túnez ha sido el más pacífico y exitoso de todos cuantos han acaecido en Oriente Medio y Norte de África. No obstante, aunque han sido muchas las sombras que han surgido en el proceso a día de hoy Túnez es más democrático que con Ben Alí. En este proceso Qatar ha tenido influencia, aunque Túnez no era una de las prioridades de Doha cuando surgieron las Primaveras Árabes. A día de hoy, el interés ha crecido ya que es la única opción que le queda tras los fracasos de Siria, Libia y Egipto.

\subsubsection{Apoyo político y diplomático}

El punto donde más se ha apreciado el apoyo qatarí en Túnez ha sido, sin el menor género de dudas, en el aspecto político. Los vínculos entre Doha y el partido Ennahda -cercano a los Hermanos Musulmanes- han marcado la relación desde los primeros momentos de la Revolución del Jazmín.

\footnotetext{
28 "The Muslim Brotherhood is the only clandestine, organized political force in Saudi society and its rise to power in Egypt has pleased its sympathizers across the Red Sea" Al Omran, Ahmend: "Saudi Arabia: A new mobilization" Gulf Analysis-ECFR, (abril 2013), p. 5.

${ }^{29}$ Líder del partido tunecino Ennahda vinculado a los Hermanos Musulmanes.

${ }^{30}$ Líder de Hamás.

${ }^{31}$ Líder del partido yemení Al-Islah.

${ }^{32}$ Priego, Alberto: "La nueva política exterior de Arabia Saudí" Documento Marco-IEEE, 20 de agosto de 2015, en

http://www.ieee.es/Galerias/fichero/docs marco/2015/DIEEEM18-

2015 NuevaPoliticaExterior_ArabiaSaudi A.Priego.pdf.
} 
Uno de los aspectos más importantes ha sido la ayuda financiera prestada directamente al partido. Gracias a esta ayuda, Ennahda ha podido afrontar los comicios en condiciones de ganarlos $^{33}$. Esta asistencia se ha podido gestionar gracias a los contactos establecidos previamente entre miembros de Ennahda -esencialmente a través de su líder Rashid Al Ghannouchi- y personalidades políticas y religiosas de Qatar. De hecho, los contactos y las visitas de miembros de ambos gobierno a Qatar o Túnez son frecuentes ${ }^{34}$. Como ejemplo de estas excelentes relaciones, el propio Emir visitó Túnez para celebrar el primer aniversario de Revolución ${ }^{35}$.

A día de hoy tras los sonados fracasos de Libia, Egipto y Siria, Túnez se ha convertido en la única baza de Qatar. Además, la moderación tunecina permite a Qatar, a pesar de todos los problemas que ha creado, mostrar la opción de la hermandad como una opción compatible con la democracia.

\subsubsection{Asistencia económica}

Si bien es cierto que la ayuda financiara qatarí en Túnez ha sido importante, no es menos cierto que, en cuantía, ha sido mucho menor que en el caso de Egipto o Libia. En todo caso, tras la caída de Ben Alí las relaciones comerciales entre Qatar y Túnez se dispararon.

Es difícil calcular cuando dinero está aportando Qatar a Túnez, pero algunas fuentes (Wilson Center) apuntan a una cifra que rondaría los 1.500 millones de dólares. En todo caso no toda esta ayuda económica es a fondo perdido. Algunas prestaciones económicas se han puesto en marcha en forma de préstamo con un interés del 2,5\% de interés. También hay que señalar que Qatar ha evitado la bancarrota de Túnez aportando importantes depósitos al Banco de Túnez. Tras la experiencia de Libia y Egipto, Qatar está tratando de maximizar el proceso tunecino y por ello considera que la paz social y económica son puntos básicos para su éxito. No se puede olvidar que el éxito del caso tunecino puede contribuir a la consecución de los fines de política exterior qatarí anteriormente mencionados. Para ello, entre otras muchas cosas Qatar está financiando multitud de proyectos a través de una fundación de apoyo a los mártires Tunisia's Martyr Fund. Además, a nivel empresarial Doha está realizando importantes adquisiciones de compañías tunecinas como el principal operador de móvil o el principal banco que ahora se llama Tunisia Qatari Bank. Junto a estas adquisiciones también está invirtiendo en infraestructuras como el complejo turístico Tozeur o la refinería de Skhira. Esta refinería no solo resultó importante para la economía tunecina sino también para la libia ya que buena parte del petróleo que allí se refinaba procedía del país vecino.

Estos ejemplos no son más que muestras del interés qatarí por la estabilidad política, económica y social en Túnez.

\subsubsection{Ayuda militar}

A nivel militar, la ayuda a Túnez ha sido mucho menos significativa que otros casos como el de Libia o Siria. La explicación la tenemos que buscar en el carácter pacífico de la transición tunecina y la importancia relativa del ejército. Sin embargo y a pesar de esto, desde la caída de Ben Alí se han incrementado los vínculos militares entre los dos estados. De hecho, fruto de este acercamiento se ha firmado dos importantes acuerdos en materia militar ${ }^{36}$.

\footnotetext{
33 "Qatar finances Ennahda's rule in Tunisia with $\$ 500$ million", Middle East On Line, 23 de noviembre de 2013 http://www.middle-east-online.com/english/?id=62816.

34 "Al Ghannouchi recently visited Qatar Orphan Foundation (Dhreima)", The Penninsula, 10 de julio de 2015 http://thepeninsulaqatar.com/news/middle-east/347339/ennahda-chief-defends-tunisia-emergency

35 "Sheikh Hamad bin Khalifa al-Thani came to Tunisia to celebrate Tunisia's revolution with interim President Moncef Marzouki", Tunis Time, 3 noviembre de 2013.

36 "Qatar, Tunisia sign two key military pacts", Qatar News Agency ,20 de noviembre de 2012.
} 
A pesar la aparente buena relación entre los dos estados, existen importantes críticas a la política de Qatar. Importantes sectores de la sociedad civil tunecina no ven con buenos ojos la mano del emirato, que temen la radicalización de la vida diaria tunecina. Las redes sociales han sido el terreno de protesta para estos sectores más críticos. En ellas se han desarrollado campañas como "Campaign to insult the state of Qatar' que contaba con 23.000 seguidores en Facebook a las pocas horas de lanzarse. El propio presidente de Túnez hizo un llamamiento a la población a acabar con esta actitud, coincidiendo con una ceremonia de entrega de fondos por parte de las autoridades qataríes ${ }^{37}$.

\subsection{Libia}

Si hay un lugar donde Qatar ha significado a favor del cambio, ese ha sido Libia. El posicionamiento de Doha en favor de los rebeldes ha sido tal, que algunos autores hablan incluso de una revisión de su política exterior ${ }^{38}$. El gobierno de Qatar ha aportado dinero, armas, entrenamiento, pero por encima de todo esto apoyo diplomático a las fuerzas que derrocaron al régimen de Gadafi. Por ello la principal Plaza de Trípoli, la plaza de Argelia, fue renombrada como la Plaza de Qatar ${ }^{39}$.

Dentro del proceso de cambio político que se ha vivido en Libia, han resultado cruciales los apoyos prestados por Qatar a personas concretas vinculadas con la Hermandad. Este aspecto será abordaremos más adelante con cierto detalle.

\subsubsection{Apoyo político y diplomático}

Desde que se iniciaron las protestas en Libia, Qatar se convirtió en el principal apoyo político y diplomático de la oposición a Gadafi. Para ello, desde el Ministerio de Asuntos Exteriores de Qatar se impulsaron dos ambiciosas y arriesgadas acciones diplomáticas que tenían como objetivo final el derrocamiento del régimen de Gadafi.

a) El apoyo a la intervención internacional liderada por la OTAN tanto en la ONU y como en la Liga Árabe.

b) El reconocimiento internacional del Consejo Nacional de la Transición como gobierno legítimo de Libia.

a) Apoyo internacional en las organizaciones internacionales: El Ministerio de Asuntos Exteriores de Qatar se erigió en el máximo defensor de la intervención armada internacional aunque hay que decir que en los meses inmediatamente anteriores Doha no se había mostrado como un enemigo de Gadafi. Sin embargo, la rebelión que se inició el 15 de febrero en Benghazi no podría haberse consumado sin el apoyo diplomático de Qatar ya que, desde Doha se allanó el terreno para la aprobación de la Resolución 1973 y la posterior intervención de la OTAN tanto en el mundo árabe, como en las organizaciones internacionales regionales. Este esfuerzo internacional de Qatar buscaba un claro objetivo: reforzar ese rol internacional diferenciado que le asegurara su seguridad aportando legitimidad a una intervención de corte occidental que iba contra un estado Árabe ${ }^{40}$.

\footnotetext{
37 "Marzouki Asks Tunisians to Respect Qatar; Many Loudly Disobey" Tunisia live, 23 de abril 2013 http:/www.tunisia-live.net/2013/04/12/marzouki-asks-tunisians-to-respect-qatar-many-loudlydisobey/\#sthash.NovyOD0B.dpuf.

${ }^{38}$ Echagüe, Ana: "Emboldened yet vulnerable: the changing foreign policies of Qatar and Saudi Arabia" FRIDEWorking paper $\mathrm{n}^{\circ} 123$ July 2014, p. 7. Khatib, Lina: "Qatar's Foreign Policy: the limits of pragmatism", International Affairs, vol. 89, no. 2, 2013, p. 429.

${ }^{39}$ Coates-Ulrichsen, K. (2014): Qatar and the Arab Spring, Hurst \& Company, London, p. 128.

40 "the Libya crisis offered an opportunity for Qatar to align its support for the protection of human rights and democratic expression in a manner that resonated powerfully with the (Western-led) international community" Coates-Ulrichsen, op. cit., p. 124.
} 
Para consumar esta acción, cabe destacar las gestiones llevadas a cabo por el entonces Primer Ministro Hamad bin Jassim Al-Thani. Gracias a estas acciones Doha asumió la presidencia en la Liga Árabe, aun cuando no le correspondía y de este modo pudo suspender a la Libia de Gadafi como miembro de dicha organización.

b) Reconocimiento Diplomático Internacional del Consejo Nacional de la Transición: E1 reconocimiento internacional del Consejo Nacional de la Transición se alcanzó a través de los canales tradicionales y no tradicionales de la diplomacia. En lo que a la diplomacia tradicional se refiere, Qatar fue el segundo estado del mundo, en reconocer al Consejo Nacional de Transición como gobierno legítimo de Libia sólo después de Francia. Más allá de poder presumir de haber sido el segundo del mundo, lo que verdaderamente fue significativo es su condición de estado árabe ${ }^{41}$. Sin embargo, ese reconocimiento nunca se hubiera alcanzado de no haber utilizado los medios no-tradicionales de la diplomacia, sobre a través de las acciones de la televisión Al Jazeera ${ }^{42}$ que jugó un papel muy importante en la difusión del mensaje. Junto a la información que difundía Al Jazeera sobre la situación de Libia, hay que destacar a la televisión rebelde libia Al Ahrar, que emitiendo desde Doha, influyó enormemente al colocar la cuestión libia en el centro de la agenda internacional.

Por último y unido a lo anterior, hay que mencionar el esfuerzo diplomático multilateral desarrollado por Qatar a través del Grupo de Contacto de Libia. Gracias a la influencia de este grupo de estado la causa rebelde no solo se hizo más conocida a nivel internacional, sino que permitió coordinar la ayuda internacional frente a un Gadafi que estaba bien organizado.

De este modo, usando tanto la diplomacia tradicional como la no tradicional, Qatar consiguió desarrollar, al menos temporalmente, un rol que solo ellos pueden lograr: erigirse como un actor internacional con características únicas que aseguraba así sus objetivos en política exterior (notoriedad y supervivencia).

\subsubsection{Apoyo militar}

En el plano militar Qatar se ha mostrado profundamente generoso con los rebeldes libios. Este apoyo fue incluso reconocido explícitamente por el Jefe del Estado Mayor qatarí ${ }^{43}$. Para ello, de nuevo resultaron fundamentales los contactos previos establecidos por Qatar con radicales vinculados directamente o indirectamente a los Hermanos Musulmanes. Entre estos contactos podemos destacar a los siguientes como los más significativos:

i) Abdel Hakim Belhaj: Vinculado a Al Qaeda y detenido en 2004 fue liberado gracias a la mediación de Qatar que participó en un programa de "des-radicalización" dirigido por Saif Al Islam Gadafi ${ }^{44}$. Posteriormente dirigió a las Brigadas de los Mártires del 17 de febrero que participó en la toma de Trípoli.

ii) Ali e Ismail al-Sallabi: hijos de un banquero libio vinculado a la hermandad, ambos han estado directamente vinculados a Qatar. Ali, es un clérigo formado en Doha bajo la

\footnotetext{
41 "Qatar gave support to opposition forces against Libya's Colonel al Qaddafi and was the first Arab state to officially recognize the opposition after that", en Roberts, David "Qatar's Foreign Policy Roberts, David: "Qatar's Foreign Policy Adventurism" Foreign Affairs (Snapshot), 25 junio 2013.

42 "It acted as an interlocutor for the Arab League and Arab states who were pushing for international intervention in Libya, not only through diplomacy, but also through public diplomacy, namely through the Qatar-based television station al-Jazeera, which became the leading voice of the Arab Spring in 2011", en Khatib, Lina: "Qatar's involvement in Libya: A delicate balance", World Peace Foundation, 7 de enero de 2013. Disponible en http://sites.tufts.edu/reinventingpeace/2013/01/07/qatars-involvement-in-libya-a-delicate-balance/.

43 Black, Ian: "Qatar admits sending hundreds of troops to support Libya Rebels", The Guardian, 26 octubre de 2011.

${ }^{44}$ Black, Ian: "The Libyan Islamic Fighting Group - from al-Qaida to the Arab spring", The Guardian, 5 septiembre de 2011.
} 
protección de Yusuf Qadarawi, que resultó fundamental para canalizar la ayuda militar de Qatar a los rebeldes libios ${ }^{45}$. Su hermano Ismail dirigió al grupo Tajamauu Saraya alThuwar.

iii) Jalal al-Dugheily: actuó como Ministro de Defensa del CNT y por esta condición en julio de 2011 se reunió en Doha con el Jefe del Estado Mayor de Qatar Mr. Attiyah con el fin de coordinar la ayuda financiera y militar a los rebeldes ${ }^{46}$.

En cuanto al apoyo militar, podemos dividir el apoyo militar de Qatar a los rebeldes libios en tres puntos fundamentales, sin los cuales el derrocamiento de Gadafi hubiera sido simplemente imposible.

a) Transferencia de armas.

b) Adiestramiento de fuerzas.

c) Envío de fuerzas sobre el terreno.

a) Transferencia de armas: Es conocido por todo el mundo que Qatar ha contribuido a las fuerzas anti-Gadafi enviando importantes remesas de armas. Aunque el gobierno de Doha no lo ha reconocido -habla de material bélico defensivo- se calcula que Qatar podría haber enviado hasta 20.000 toneladas de armas a Libia ${ }^{47}$, violando las resoluciones del Consejo de Seguridad. Por ello, hay que decir que la transferencia de armas a los rebeldes libios fue una pieza fundamental para el derrocamiento de Gadafi. En este punto, Qatar se convirtió en un actor único que facilitó el rearme de los rebeldes y por lo tanto la caída de Gadafi.

b) Adiestramiento de fuerzas. Otra de las aportaciones qataríes a la caída de Gadafi ha sido el entrenamiento de las fuerzas rebeldes. Qatar cumplió un rol fundamental en el adiestramiento de las fuerzas rebeldes que se enfrentaron la guardia del Coronel Gadafi. Para ello, fuerzas especiales de Qatar entrenaron directamente a la infantería rebelde en las montañas de Nafusa, en Libia Oriental. Sin embargo, la cooperación no quedó aquí y fuerzas libias fueron enviadas a Doha para recibir adiestramiento militar especial en los cuarteles del ejército qatarí ${ }^{48}$. Este adiestramiento sirvió para organizar a unas fuerzas poco disciplinadas y con escasa formación militar.

c) Envío de fuerzas sobre el terreno. Además de los aviones Mirage $2000^{49}$ qataríes que se integraron en la fuerza de la OTAN (Operation Unified Protector), Qatar envió fuerzas terrestres a combatir en Libia contra Gadafi, sobrepasando las resoluciones del Consejo de Seguridad de Naciones Unidas. Este hecho sí que fue reconocido por el Jefe del Estado Mayor qatarí, Hamad bin Ali Al-Athiya quien declaró "we were among them and the numbers of

\footnotetext{
45 "Qatar funneled much of its aid through Ali al-Sallabi" Dagher, Sam and Levinson, Charles: "Tiny Kingdom's huge role in Libya draws concern" The Wall Street Journal, 17 October 2011.

46 "Armed forces chief holds talks", Gulf Times, 6 July 2011.

${ }^{47}$ Dagher, Sam and Levinson, Charles: "Tiny Kingdom's huge role in Libya draws concern", The Wall Street Journal, 17 octubre de 2011.

48 "The Qatari military even brought Libyan fighters back to Doha for special exercises", en Roberts, David B: "Behind Qatar's Intervention in Libya. Why was Doha such a strong supporter of the Rebels?" Foreign Affairs (Snapshot), 28 September 2011.

49 "The dispatch of our four Mirage 2000 fighters to Crete gave the military operations the crucial Arab support needed to overcome nagging western doubts about the campaign", en Coates-Ulrichsen, Kristian: "Qatar and the Arab Spring", Open Democracy, April 2011, en https://www.opendemocracy.net/kristian-coates-ulrichsen/qatarand-arab-spring.
} 
Qataris on the ground were hundreds in every regions ${ }^{50 "}$ Estas fuerzas además de combatir, sirvieron de enlace entre la OTAN y los rebeldes libios. De nuevo, esta tarea se pudo llevar a cabo de forma exitosa gracias a las redes establecidas entre Qatar y los Hermanos Musulmanes libios, contactos que se remontan varios años atrás.

A modo de resumen, podemos afirmar que la ayuda militar qatarí fue fundamental para derrocar a Gadafi. Esta ayuda se materializó en transferencia de armas, adiestramiento de fuerzas así como el combate directo de fuerzas qataríes sobre el terreno libio. En todo caso, la asistencia qatarí nos muestra la particularidad de Doha a nivel internacional.

\subsubsection{Asistencia económica}

El tercer pilar de la ayuda qatarí es la asistencia económica. Se calcula que Qatar habría aportado a los rebeldes unos $400 \$$ millones de dólares en ayuda económica no militar. Esencialmente estamos hablando de depuradoras, desalinizadoras de agua, calentadores de gas y bienes de primera necesidad.

Sin embargo, Qatar no solo aportó ayuda de forma directa sino que también jugó un papel fundamental ayudando a los rebeldes a situar en el mercado del petróleo que se almacenaba en los puertos del Este de Libia ${ }^{51}$. Ambas acciones resultaron fundamentales para el derrocamiento del régimen de Gadafi.

Para concluir este apartado, podemos afirmar que Qatar jugó un papel fundamental en la caída de Gadafi. Su apoyo diplomático, económico y militar permitió alterar el orden regional en el Norte de África, y si bien luego la seguridad de Libia se ha deteriorado, en los primero momentos de la revolución permitió a Qatar desarrollar su política exterior diferenciada.

A día de hoy, Qatar sigue manteniendo discrepancias con Arabia Saudí sobre quién debe gobernar Libia. Mientras que Doha y Ankara apuestan un partido vinculado a los Hermanos Musulmanes "Amanecer Libio", Riad y El Cairo son partidarios del Parlamento de Tobruk y las fuerzas del General Khalifa Haftar. En todo caso, las acciones qataríes en Libia nos muestran como Doha ha buscado diferenciarse de la política de otros estados.

\subsection{Egipto}

Las relaciones entre Qatar y Egipto no han sido ni mucho menos fáciles. Muchos han sido los asuntos que han generado fricción entre los dos estados, pero se podría resumir en una frase: Qatar emerge como polo de atracción del Mundo Árabe frente a un Egipto decadente que no puede mantener ese rol $^{52}$ más tiempo. Aun hoy, con unas relaciones menos malas siguen surgiendo puntos de fricción como la condena a tres años de cárcel a dos periodistas de $\mathrm{Al}$ Jazeera por su simpatía con los Hermanos Musulmanes. Este asunto será abordado más adelante.

Si nos centramos en las cuestiones que han sido más polémicas, podemos destacar los vínculos entre Qatar y Hamás, la mediación en Sudán y sobre todo el asilo que Doha brinda desde hace décadas a miembros de los Hermanos Musulmanes que huyen de la represión ejercida primero por Nasser, después Sadat y posteriormente por Mubarak.

Quizás el asunto que más ha contribuido a empeorar las ya malas relaciones entre Egipto y Qatar ha sido la actividad periodística de la cadena de televisión Al Jazeera. Desde que comenzara a emitir en Egipto (2000), la cadena qatarí ha sido vista con mucho recelo por

\footnotetext{
${ }^{50}$ The Daily Start (Lebanon), 26 octubre de 2011.

${ }^{51}$ Coates-Ulrichsen, K. (2014): Qatar...op. cit., p.126.

${ }^{52}$ Kamrava, Mehran (2013): Qatar. Small State, Big Politics. Cornell University Press, Ithaca \& London, p. 29.
} 
parte de las autoridades egipcias ${ }^{53}$. El punto culminante fue el ya mencionado asunto de los tres periodistas de Al Jazeera que fueron encarcelados en Egipto acusados de complicidad con los Hermanos Musulmanes ${ }^{54}$. Uno de ellos, de nacionalidad australiana, fue liberado a comienzo del verano pasado, mientras que los otros dos, han sido recientemente condenados a tres años de cárcel.

No obstante, y a pesar de que en Egipto los vínculos con los Hermanos Musulmanes eran muy fuertes, la caída de Mubarak cogió por sorpresa a los qataríes, quienes además en las semanas previas a la revolución habían rebajado el tono de crítica hacia el gobierno egipcio. La mejor ilustración de esa entente-cordiale la encontramos en las palabras de un diplomático egipcio tras una reunión con el primer Ministro de Qatar HBJ "We accept the role that Qatar wants to play as a growing political mediator in the Middle East, and Qatar accepts to notify and cooperate with $\mathrm{s}^{55}$ " Sin embargo, al igual que ocurrió en el caso de Libia, la revolución no estaba en las cabezas de los líderes qataríes. No obstante, su rápida reacción permitió a Doha jugar un rol protagonista en el proceso con el fin de cumplir sus objetivos en política exterior.

\subsubsection{Apoyo político y diplomático}

En el caso de Egipto, el apoyo mostrado por Qatar fue sobre todo político y diplomático. Precisamente por ello, Doha se convirtió en el valedor internacional del gobierno del Presidente Mursi, jugándose incluso su relación con los Estados Unidos. A diferencia de los casos de Siria y de Libia, donde se produjo un cambio de régimen y, por tanto, fue necesario el reconocimiento internacional, en Egipto el cambio fue solo político lo que propició que los esfuerzos diplomáticos tuvieron otra dimensión.

Un punto fundamental del apoyo político de Qatar al gobierno de los Hermanos Musulmanes fue la gestión de las relaciones con los Estados Unidos. El decreto de noviembre 2012 que incrementaba los poderes del presidente, no fue bien visto por los Estados Unidos. En ese punto, Qatar usó toda su influencia en Washington para evitar que Estados Unidos viera al nuevo Egipto como una amenaza regional. Para afianzar más este apoyo, el propio Emir de Qatar realizó dos visitas en las que se entrevistó personalmente con el Presidente Mursi, mostrando así su apoyo al nuevo mandatario egipcio.

El caso de Egipto es donde se ha visto de forma más clara el pulso diplomático entre Doha y Riad. Frente a la ilusión desatada en Qatar por la victoria de la hermandad, Arabia Saudí lo vio como una amenaza directa y por ello, comenzó a trabajar para su derrocamiento. De hecho, Arabia Saudí temió que la llegada al poder de los Hermanos Musulmanes en Egipto supusiera una amenaza para su estabilidad política. Para evitar una situación similar, el gobierno de Riad aprobó varios documentos que impulsaban leves acciones de liberalización política. En esta línea, Riad aprobó jugosas y generosas ayudas públicas para evitar que los Hermanos Musulmanes pudieran encontrar público en Arabia Saudí ${ }^{56}$.

\footnotetext{
${ }^{53}$ Seib, Philip (2008): The Aljazeera Effect. How the new Global Media are reshaping world of politics. Washington D.C., Potomac Books, pp. 144-145.

54 "Egypt court sentences Al Jazeera journalists" Al Jazeera, 23 de junio de 2014, en http://www.aljazeera.com/news/middleeast/2014/06/egypt-finds-al-jazeera-journalists-guilty201462373539293797.html

55 "Egypt and Qatar pursue new economic and political cooperation", Arhamonline, 11 de diciembre de 2010, en http://english.ahram.org.eg/NewsContentP/1/1663/Egypt/Egypt-and-Qatar-pursue-new-economic-and-politicalaspx.

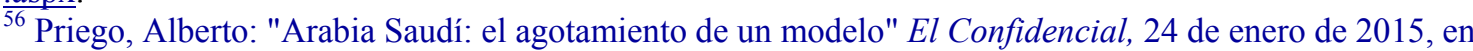
http://www.elconfidencial.com/mundo/2015-01-24/arabia-saudi-el-agotamiento-de-un-modelo-deestado 629144/.
} 
Sin embargo, a pesar del apoyo mostrado por Qatar al nuevo gobierno egipcio y de los esfuerzos diplomáticos desarrollados por el propio Mursi ${ }^{57}$, los Hermanos Musulmanes no pudieron mantenerse en poder. Gracias al apoyo de Arabia Saudí - valedor del golpe de estado de Al Sisi- los Hermanos Musulmanes fueron desalojados del poder y sus miembros encarcelados y en algunos casos condenados a muerte. El punto de inflexión para el gobierno de Mursi y por lo tanto para Qatar llegó en la primera semana de julio. En esa semana en la que se produjeron los enfrentamientos más violentos, Doha mostró de forma clara su apoyo a los Hermanos Musulmanes. Así, Qatar condenó la muerte de 55 miembros de la hermanad a manos del ejército y abogó por la liberación del Presidente Mursi. Como represalia a ese apoyo explícito a los Hermanos Musulmanes, el nuevo gobierno egipcio cerró la oficina de Al Jazeera en El Cairo y detuvo a los tres periodistas anteriormente mencionados. Qatar reaccionó retirando a su embajador en enero de $2014^{58}$ y desde entonces las relaciones se han ido deteriorando.

El fracaso del laboratorio egipcio, elimina la posibilidad de que Qatar pueda tener influencia mayor en el país del Nilo, al menos, en un futuro cercano. En este sentido Qatar sale claramente derrotado del escenario egipcio, sobre todo tras la llegada al poder del Presidente Al Sisi, que pudo convertirse en el jefe del ejecutivo gracias a las presiones ejercidas por Arabia Saudí y Estados Unidos ${ }^{59}$. Esta derrota quedó escenificada por el relevo de Qatar de la Franja de Gaza cuando el Reino apoyó el alto el fuego presentado por Al Sisi dejando en agua de borrajas los esfuerzos llevados a cabo por Doha desde 2007. Así el eje Al Sisi-Riad sustituía al eje Mursi-Doha.

Sin embargo, la situación tampoco está siendo sencilla para el militar egipcio ya que su capacidad para transmitir estabilidad se encuentra en entredicho por la continuidad de los atentados y hechos como la dimisión del bloque de su gobierno acosado por la corrupción o el asesinato de varios turistas mexicanos que fueron confundidos con terroristas del Estado Islámico.

\subsubsection{Apoyo militar}

Desde el punto de vista militar, la cooperación entre Doha y los Hermanos Musulmanes no fue tan significativa como en el caso de Libia aunque merece ser destacada. La explicación responde a dos causas. Por un lado, Egipto ha venido cooperando con los Estados Unidos en esta cuestión, y por tanto, el ejército es un poder en sí mismo y no necesita ayuda exterior. Es más, el propio ejército fue uno de los poderes que más se opusieron al cambio propuesto por Mursi. La segunda causa es que en Egipto no se ha producido un cambio de régimen como sí que ha ocurrido en Libia. Por lo tanto, no le vamos a dedicar más atención a este punto.

\subsubsection{Apoyo económico}

Junto con el apoyo político, la asistencia económica ha sido la línea más clara de Qatar en el Egipto de los Hermanos Musulmanes. Es difícil saber verdaderamente cuánto dinero ha invertido Qatar en Egipto. En un primer momento el Primer Ministro qatarí anunció una donación de 2.500 millones de $\$^{60}$ y 2000 millones en depósitos para el banco central. A esta cantidad habría que sumar unos 500 millones de $\$$ en becas. Poco tiempo después, en una de las visitas realizadas por el Emir a Egipto, se anunció la compra de 3.000 millones de $\$$ en bonos así como asistencia en el suministro de gas para evitar los cortes provocados por el

\footnotetext{
${ }^{57}$ Mursi realizó su primer viaje oficial a Arabia Saudí.

58 "Qatar withdrew its ambassador in January" The Middle East, February 2014.

${ }^{59}$ Echagüe, Ana: "Emboldened yet vulnerable: the changing foreign policies of Qatar and Saudi Arabia" FRIDEWorking paper, $\mathrm{n}^{\circ} 123$, July 2014, p. 8.

${ }^{60}$ Haykel, Bernard: "Saudi Arabia and Qatar in a Time of Revolution", Gulf Analysis Paper, (February 2013), p.6, en http://csis.org/publication/gulf-analysis-paper-saudi-arabia-and-qatar-time-revolution.
} 
aislamiento internacional. En general, se calcula que Qatar podría haber aportado unos 8.000 millones de dólares al régimen del Presidente Mursi, algo que sin embargo no le ha servido para lograr su objetivo: incrementar su influencia en el Mundo Árabe en general y en Egipto en particular. En todo caso, esta política sí que sirve para mostrar cuál fue el nivel de compromiso de Doha con el régimen del Presidente Mursi.

A modo de curiosidad hay que decir que tras la llegada al poder del General Al Sisi, Egipto devolvió los 2.000 millones de \$ que Qatar había depositado en el Banco Central de Egipto $^{61}$. Por su parte, Arabia Saudí sí que ha asistido económicamente al Egipto del General Al Sisi lo que certifica el cambio de influencia en Egipto y la derrota de Qatar.

Para concluir este apartado, podemos decir que si bien es cierto que Qatar ejerció influencia en Libia de forma monopolística, en Egipto Riad ha tratado de limitar y eliminar cualquier forma de influencia procedente del emirato. A día de hoy Arabia Saudí ejerce una mayor influencia en Egipto, y por su parte, Qatar ha aceptado un rol no solo secundario sino subordinado lo que supone el fracaso de los postulados iniciales qataríes.

\subsection{Siria}

Siria ha sido el escenario donde de forma más clara, han chocado los dos colosos del Golfo Pérsico: Qatar y Arabia Saudí. Si bien es cierto que, al menos ahora, los dos estados tienen un interés común, acabar con Al Assad, cada uno tiene sus preferencias respecto del grupo que debe suceder al dirigente sirio. Así, Siria se ha configurado como el escenario donde, de forma más explícita, se ha mostrado el enfrentamiento entre Arabia Saudí y Qatar.

Como hemos visto anteriormente, Qatar no ha sabido entender el escenario Sirio y por ello ha recibido un golpe significativo en sus aspiraciones regionales. Así, lo ocurrido en Siria ha dejado en entredicho su capacidad de influir en Oriente Medio. La explicación a esta situación la encontramos en un error de cálculo propiciado por considerar que Siria y Libia eran casos similares cuando son realidades diferentes. Así, podemos destacar los siguientes elementos como los puntos fundamentales del fracaso qatarí en Siria.

1) Ausencia de grupos afines (Hermanos Musulmanes): Siria no era un territorio tan propicio para Qatar como lo fue Egipto o Libia ya que la presencia de los Hermanos Musulmanes era casi testimonial. El motivo no es otro que la represión ejercida a comienzo de los años 80 por Hafez Al Assad. Por lo tanto, Qatar no contaba con una de sus herramientas fundamentales en política exterior lo que hacía más complicado la consecución de sus fines.

2) Intereses estratégicos internacionales: Siria es un uno de los centro neurálgicos de Oriente Medio y es precisamente allí donde confluyen buena parte de las divisiones de la región: chítas-sunitas, árabe-israelí o confrontación Rusia-EEUU. Por lo tanto, un cambio de régimen en Siria se antojaba más complicado que en otros lugares como Túnez o Libia ya que implicaba una nueva configuración regional de Oriente Medio

3) Apoyos Internacionales: Si bien es cierto que la Libia de Gadafi se encontraba internacionalmente aislada, el régimen de Al Assad contaba con importantes apoyos internacionales, entre otros Rusia e Irán. Por ello, las posibilidades de aislar internacionalmente a Siria eran muchos menores a pesar de los esfuerzos de los líderes qataríes por lograr la condena internacional del régimen de Al Assad. Esta presión provocó un calentamiento en las relaciones entre Damasco y Doha que es percibido por Al Assad como uno de sus principales enemigos internacionales. De hecho, fue precisamente la presión ejercida desde Al Jazeera lo que provocó el ataque a la

\footnotetext{
61 "Egypt returns \$2 billions to Qatar in sign of growing tensions" Reuters, 19 September 2013.
} 
Embajada de Qatar en Damasco, paso previo para la ruptura de relaciones diplomáticas que se consumó en julio de $2011^{62}$.

\subsubsection{Apoyo político y diplomático}

Podemos destacar dos líneas de acción del gobierno de Qatar en la transición siria. Por un lado, ha buscado granjearse el apoyo de grupos opositores a Al Assad, esencialmente los Hermanos Musulmanes, mientras que por el otro, Doha ha propiciado el aislamiento internacionalmente del régimen con el fin de lograr el reconocimiento internacional de la oposición. En cierto modo no difiere en exceso de lo que ha hecho en Libia pero desde luego los resultados han sido diametralmente distintos. No obstante, existe un denominador común a ambos casos, ya que lejos de conseguir sus objetivos Qatar se ha encontrado la oposición de Arabia Saudí quien a su vez tenía intereses similares. En todo caso vamos a analizar estas dos acciones para poder evaluar cuál ha sido el papel de Qatar en Siria.

1) Captación de grupos opositores cercanos a los intereses de Qatar: Como hemos señalado anteriormente uno de los problemas que ha tenido Qatar en Siria ha sido la falta de apoyo interior. Quizás por ello, a sabiendas del escaso poder que tenían los Hermanos Musulmanes, Qatar ha tratado de acercarse a los grupos de la oposición para ganarse su confianza en un escenario post-Al Assad. Así, en agosto de 2011 Qatar apadrinó la creación del Consejo Nacional Sirio en el que la Hermandad siria ocupaba un rol destacado. Qatar logró situar como vicepresidente a Mohammed Farouk Tayfour y a Molham al Drobi como "Ministro de Asuntos Exteriores" ambos conocidos miembros de la Hermandad. Sin embargo, esta acción acabó en fracaso ya que los Hermanos Musulmanes no tienen repercusión en Siria y toda su capacidad se centra entre los exiliados. Es decir su capacidad para representar a los sirios es muy limitada ${ }^{63}$.

En una línea similar y derivado del fracaso anterior, Qatar realizó otro intento para controlar a la oposición Siria con la creación de la Coalición Nacional Siria de Revolución y Fuerzas de Oposición. En este nuevo grupo también se incluía al mencionado Consejo Nacional Sirio. Para ello, en noviembre de 2012 Qatar organizó una reunión en Doha con los líderes de las principales facciones. Ha sido precisamente dentro de este grupo donde se ha encontrado de forma más clara con la oposición de Arabia Saudí quien aprovechó el encuentro para apoyar a sus propios candidatos (primero al-Jarba y después Hadi-al-Bahra). Sin embargo los desencuentros dentro de la propia coalición no han sido solo entre Qatar y Arabia Saudí, sino también entre miembros de la propia familia Saud. Este es el caso del enfrentamiento entre el antiguo ministro de asuntos exteriores saudí, Abdulaziz al-Saud y el jefe de la inteligencia bin Sultan. Cada uno apoyaba a distintos candidatos que buscaban liderar la Coalición Nacional. Estas diferencias han supuesto un lastre para vencer al régimen de Al Assad.

En todo caso, los cambios producidos en la casa Al Saud también han propiciado una redefinición de la estrategia. La sustitución del Príncipe Bandar bin Sultan por los Príncipes bin Nayef y bin Salman hace presagiar cambios aunque todavía es pronto para hacer una evaluación. Estos cambios deberían generar un acercamiento a las posiciones de Qatar y Turquía. Este punto lo trataremos posteriormente.

2) Esfuerzos diplomáticos: En el plano diplomático el asunto de Siria ha estado profundamente influido por los acontecimientos acaecidos en Libia. El apoyo ruso al

\footnotetext{
62 "Syria accused over attacks on Saudi and Qatari embassy", BBC, 13 November 2011.

${ }^{63}$ Oweis, Khaled: "Syria's Muslim Brotherhood rise from the ashes", Reuters, 6 de mayo de 2012.
} 
régimen de Al Assad anticipaba un bloqueo en el Consejo de Seguridad por lo que Doha optó por centrar todos sus esfuerzos en otros foros en concreto en la Liga Árabe.

Sin embargo, y a sabiendas de la imposibilidad de contar con el consentimiento del Consejo de Seguridad, tanto el Emir como el Primer Ministro de Qatar no dejaron pasar la oportunidad para hacer llamamientos a la Comunidad Internacional en favor de una intervención humanitaria en Siria. ${ }^{64}$ Concretamente el Emir Hamad Bin Khalifa llegó a decir en la cadena norteamericana CBS "for such a situation to stop...some troops should go to stop the killing" aunque éstas no fueron las declaraciones más duras. El Primer Ministro, Hamad bin Jassim habló incluso de genocidio para describir lo que estaba ocurriendo en Siria ${ }^{65}$.

Como se desprende tanto de las palabras del Emir como de las del Primer Ministro, la intención de Doha era provocar el aislamiento internacional de Al Assad. Por ello, en abril de 2012 se vetó un plan de paz auspiciado por Naciones Unidas y apadrinado por Kofi Annan para iniciar un proceso de paz. Junto a esto, no debe ser olvidado el hecho de que Qatar haya reconocido a los rebeldes como gobierno legítimo de Siria. El apoyo qatarí no quedó en mero reconocimiento internacional ya que incluso, como muestra del compromiso qatarí con la oposición ${ }^{66}$, se llegó a abrir una embajada de la oposición siria en Doha.

En lo que a la Liga Árabe se refiere, la propuesta de Qatar -ostentaba entonces la presidencia de la organización- pasaba por la imposición de sanciones políticas y económicas a Siria. En una sesión extraordinaria y de emergencia marcada por la masacre ocurrida en la ciudad de Homs ${ }^{67}$. En este contexto, 18 estados árabes votaron a favor de la suspensión de Siria de la organización mientras que tres votaron en contra (Yemen, Líbano y Siria). Tan solo uno, Irak, se abstuvo. En todo caso, la iniciativa nos sirve para mostrar la estrategia diplomática de Qatar para lograr sus objetivos. Sin embargo, a día de hoy la situación sigue siendo complicada y el apoyo ruso e iraní permite que Al Assad se mantenga en el poder. Probablemente un esfuerzo internacional consensuado entre Riad y Doha hubiera sido mucho más eficaz y nos permitiría hablar de una situación completamente diferente en Siria.

\subsubsection{Apoyo militar}

En abril de 2012 se habló de que Qatar había llevado a cabo una donación de 100 millones de dólares a las fuerzas de la oposición siria a través de intermediarios libios. Esta política se ha ido haciendo más común a medida que las opciones de paz se han visto más remotas. Sin embargo, lejos de funcionar la asistencia militar se ha convertido en una muestra más del pulso entre Qatar y Arabia Saudí por el control de la oposición siria.

Los saudíes han focalizado sus envíos de armas en el Ejército Libre de Siria, a través de contactos libaneses establecidos en Turquía ${ }^{68}$. Concretamente se habla de Okab Sakr, un antiguo colaborador del Primer Ministro Hariri que estaría aprovechando la ayuda saudí para

\footnotetext{
${ }^{64}$ Coates-Ulrichsen, K. (2014): Qatar...op. cit., p. 136.

65 "What is happening in Syria is not a civil war but a genocide" CNN, 30 de octubre de 2011, en http://edition.cnn.com/2012/10/30/world/meast/syria-civil-war/.

66 "the villa is the new Syrian Arab Republic Embassy in Qatar, representing not the regimen of Bashar-Al Assad, but opponents fighting for his removal" Khalaf, Roula and Fielding-Smith, Abigail: " How Qatar seized control of the Syrian revolution", Financial Times, 17 May 2013.

${ }^{67}$ Unos días antes Siria había aceptado un Plan de Paz propuesto por la Liga Árabe

${ }^{68}$ Abouzeid, Rania: "Syria's Secular and Islamist Rebels: Who are the Saudis and the Qataris Arming?" Time, 18 September 2012.
} 
derrocar a su antiguo enemigo: Al Assad. Por su parte, Qatar estaría utilizando la ayuda de un antiguo miembro del ejército de Al Assad que habría desertado y que ahora sería clave en la distribución de la ayuda militar qatarí en Siria. Sin embargo, otras fuentes apuntan a otros nombres como Liwaa al-Tawhid o Ahfad al-Rasoul aunque estas personas también han sido señaladas como enlaces de Arabia Saudí ${ }^{69}$. Lo que resulta más claro son los receptores de la ayuda, los Hermanos Musulmanes y en menor medida al Frente Al-Nusra. El SIPRI ha apuntado a que sólo durante el periodo que va de marzo a abril de 2012 un total de 70 aviones de carga qataríes habrían aterrizado en Turquía con armas para distribuir en Siria entre los grupos de la oposición

De hecho, Qatar y Arabia Saudí no han sido los únicos suministradores de armamento a los rebeldes ya que a estos dos estados se ha unido un tercer estado del golfo: Kuwait ${ }^{70}$. El emirato ha establecido una oficina de distribución financiera y armamentística para los rebeldes sirios. Este punto, que resulta un tanto controvertido, se abordará en el siguiente apartado.

\subsubsection{Asistencia económica}

En enero de 2013, en el marco de las Naciones Unidas, se organizó en Kuwait una conferencia de donantes con el único fin de coordinar la asistencia humanitaria y financiera procedente de los estados del Golfo Pérsico. En términos absolutos se habló de una cantidad aproximada de 1.500 millones de dólares. De esta cantidad, Kuwait, Arabia Saudí y Emiratos Árabes Unidos aportarían aproximadamente 300 millones cada uno, y otros 180 millones de dólares procederían de donantes privados ${ }^{71}$.

A modo de crítica, cabe decir que a pesar de la ingente cantidad de dinero aportada por las monarquías del Golfo, y pesar de la coordinación de las Naciones Unidas, la ayuda no ha tenido el efecto que se esperaba. Duplicidades, opacidad, falta de coordinación entre los donantes son sólo algunos de los problemas que se han presentado en relación con el apoyo financiero del Golfo a Siria. Este problema no sólo ha desacreditado la capacidad de algunos estados de influir en la zona, sino que también ha perjudicado a la solución del conflicto ya que ha impedido que el conflicto estuviera equilibrado puesto que ha dividido más aún a la oposición a Al Assad, propiciando incluso enfrentamientos entre la propia oposición.

Para concluir este apartado debemos decir que a día de hoy la situación en Siria es muy complicada y se encuentra lejos de solucionarse. La intervención militar de Rusia con su propia agenda ha complicado aun más la situación.

\section{Conclusiones}

A modo de conclusión, podemos afirmar que las Primaveras Árabes han servido para mostrar de forma clara la decadencia en la influencia Occidental en la zona de Oriente Medio y Norte de África. A nivel multilateral, la Política de Nueva Vecindad ha resultado ser un completo desastre para la influencia de la UE en el norte de África. A nivel bilateral, potencias como Reino Unido, Francia o Estados Unidos tampoco han sabido mantener una aproximación sostenible y por ello, se han visto desplazadas por la emergencia de otros actores con una mayor cercanía cultural. Concretamente me estoy refiriendo a estados de mayoría musulmana

\footnotetext{
${ }^{69}$ Khalaf, Roula and Fielding-Smith, Abigail: "How Qatar seized control of the Syrian revolution" Financial Times, 17 May 2013.

70 "Money, guns flowing from Kuwait to Syria's most radical rebel factions" Fox News, 11 July 2013, en http://www.foxnews.com/world/2013/07/11/money-guns-flowing-from-kuwait-to-syria-most-radical-rebelfactions/.

${ }^{71}$ Coates-Ulrichsen, K. (2014): Qatar...op. cit., p. 141.
} 
como Turquía, Arabia Saudí y sobre todo Qatar. Este pequeño emirato ha visto su oportunidad en la inestabilidad política que ha sufrido Libia, Egipto, Siria y Túnez. El desencanto con los gobiernos republicanos nacionalistas, la complicidad de Occidente unido a otros condicionantes socio-económicos como la desigualdad, las altas tasas de desempleo o la corrupción han provocado la caída de regímenes no democráticos que llevaban décadas en el poder. La evolución política de estos regímenes ha sido variada. Si bien Túnez se puede decir que políticamente ha mejorado, Egipto se ha convertido en un estado aún más autoritario. Mención especial merecen Siria y Libia que tras las Revoluciones Árabes se han visto sumergidos en un caos que impide que haya tan si quiera gobierno que controle todo el territorio.

En este mar de incertidumbres Qatar ha tratado de afianzar los pilares de su política exterior: buscar la diferenciación respecto de otros estados para asegurar su propia supervivencia. Por ello, Doha ha tratado de acercarse a lo que consideraba la fuerza del cambio con un resultado más que cuestionable. Si bien es cierto que en los primeros momentos cosechó éxitos notables en Libia, Egipto y Túnez, a día de hoy su posición e influencia ha decaido. En buena medida, esta situación se debe a que Arabia Saudí no está dispuesta a renunciar a su papel de potencia regional y por ello ha tratado de competir con Qatar en la lucha por convertirse en la referencia del Mundo Árabe.

Si bien es cierto que en todos los casos se aprecia esta competencia, quizás sea en Egipto y en Siria donde es más clara. En Egipto, si bien Qatar resultó básico para la llegada al poder de los Hermanos Musulmanes, Arabia Saudí ha sido un actor decisivo para que Al Sisi esté al frente del país. En Siria la situación es distinta ya que ambos coinciden en la necesidad de acabar con Al Assad aunque no comparten la idea del grupo que debe gestión el futuro de Siria. Aquí la posición de Arabia Saudí parece mejor, ya que Qatar basa su estrategia de influencia en la presencia de los Hermanos Musulmanes y como es sabido en Siria ésta es muy débil. No obstante, un acuerdo sobre el futuro de Siria puede abrir las puertas a la estabilización de Libia y esto parece estar un poco más cerca ya que los nuevos dirigentes qataríes y saudíes están más cerca del acuerdo que los anteriores. Más allá de la propia personalidad del Rey Salman y del Emir Tamim, la coyuntura regional está propiciando un acercamiento de las posturas. Sobre todo hay dos fenómenos que está favoreciendo el citado acercamiento: la emergencia del ISIS como amenaza regional y ascenso de Irán como potencia que compite por la hegemonía en Oriente Medio, apoyado claramente en Rusia.

La primera muestra de este entendimiento lo tenemos en la solución a la crisis de los embajadores del Consejo de Cooperación del Golfo. Varios estados entre ellos Arabia Saudí, EAU y Bahréin habían retirado a su embajador de Doha por discrepancias con Qatar. A finales de 2014 la situación no solo se solucionó sino que además la cumbre se celebró en Doha $^{72}$ donde se aprobaron medidas como la creación de una fuerza naval conjunta con base en Bahréin y una fuerza policial de frontera. La primera medida está destinada a frenar el expansionismo de Irán mientras que la segunda a controlar los ataques del Estado Islámico.

Aunque en principio no es objeto de este artículo no quería dejar de mencionar una consecuencia directa de las Primaveras Árabes: una tenue liberalización económica en los estados del Golfo. En los próximos meses Arabia Saudí, EAU, Qatar y Omán van a celebrar procesos electorales. Este hecho en muchos casos en una novedad en sí mismo pero lo que es verdaderamente novedoso en la ampliación del derecho de sufragio. En Arabia Saudí las mujeres podrán votar por primera vez en las elecciones municipales. En EAU se amplía el

\footnotetext{
72 "Disagreements over these policies among the Gulf states eventually prompted Saudi Arabia, the UAE and Bahrain to withdraw their ambassadors from Doha in March 2014", en Barakat, Sultan: "Qatari Mediation. Between Ambition and Achievement" Foreign Policy - Brookings, 12 November 2014.
} 
cuerpo de electores y en Qatar están previstas elecciones municipales para mayo de 2016. Todos estos procesos son consecuencia directa de la presión ejercida por los procesos de liberación política vividos en Túnez, Libia, Egipto y Siria.

En todo caso, y como conclusión principal de este artículo hay que ser conscientes que tanto Oriente Medio como el Norte de África han dejado de ser territorios de influencia principal de los estados occidentales y que nuevos actores, con una mayor cercanía cultural y religiosa, e influencia económica comienzan a ejercer una influencia poderosa en la región. Estos actores, sin embargo, mantienen una rivalidad que afecta a la eficacia de sus políticas hacia la región. 